\title{
Three-dimensional quantitative analysis of testicular tissue sections of sprague-dawley rat: the after treatment responses to varying doses of Momordica charantia
}

\author{
Yama Oshiozokhai Eboetse, MBBS., MSc., PhD., ${ }^{1}$ Amah Chyke Ifeanyi, MBBS., MSc., ${ }^{1}$ \\ Oyebadejo Samson Adewale, BMLS/AIMLS., MSc., ${ }^{1}$ Noronha Cressie Carmel, MBBS., \\ Ms., ${ }^{1}$ Saalu Linus Chai, MBBS., MSc., PhD., ${ }^{2}$ Okanlawon Abayomi Olugbenga, MBBS., \\ M.Phil., PhD. ${ }^{1}$ \\ ${ }^{1}$ Department of Anatomy, Faculty of Basic Medical Sciences, College of Medicine of the \\ University of Lagos, Idi-Araba Lagos, Nigeria. \\ ${ }^{2}$ Department of Anatomy, College of Medicine, Lagos State University, Ikeja Lagos, Nigeria. \\ ABSTRACT
}

Background: In our previous study we scrutinized the intrinsic worth of graded doses of Momordica charantia (MC) seed extract as an antispermatogenic agent in Sprague-Dawley (S-D) rats. The corresponding histological quantification of the testicular responses to the extract is yet to be established. Objective: To investigate quantitatively using simple stereological tools the testicular reactions of S-D rats administered graded doses of $M$. charantia seed extract. Methodology: Twenty adult male S-D rats, weighing 110-214 g were assigned randomly into four groups (I-IV). Animals in groups I, II and III received varying oral doses of 15, 25 and $50 \mathrm{mg} / 100 \mathrm{~g} \mathrm{~b} . \mathrm{w} /$ day of ethanolic seed extract of MC, respectively. Group IV rats (control) were fed an equal volume of physiological saline. The duration of treatment for both extract and physiological saline was 56 days. The animals were sacrificed and the testes processed for histometry (measurement of tissues). Stereological parameters estimated include; testicular volume and weight, tubular diameter, cross sectional area of seminiferous tubules, number of profiles per unit area, length density and numerical density of the seminiferous tubules. Results: Showed a dose dependent decrease in the mean testicular volume and weight, seminiferous tubular diameter and cross sectional area. However there was an increase in the number of profiles per unit area, length density and numerical density of the seminiferous tubules.

Conclusion: Histometric data supports a dose-related deleterious effect on the testes of S-D rats treated with Momordica charantia seed extract. This also depicts a dose related antifertility potential of the extract.

Keywords: Stereological tools, Rat, Histometry, Testes

\section{INTRODUCTION}

The surface area of an interface per unit reference volume is known as surface density or specific surface (Howard and Reed, 2005). It is a useful measure of surface sensitive processes. If this surface of interest is implanted in a matrix, then the only way to make an image of the interface is to take a polished plane section through the object. The interface is seen as a boundary with dimension L1, although the parameter of significance is the area with dimension L2 on a cross-section. An evident diminution in measurement from L2 to $L 1$ has been initiated by the sectioning process. The parameter of interest is not directly observable on the section. How can the lengths $L 1$ of the observed boundaries be related to the area L2 of the surfaces of interest? (QuanToxPath, 1998). This specific question usually faced by Histologists, Material scientists, Geologists and Anatomists can simply be resolved in practice by applying stereology a branch of applied mathematics and probability theory. Stereology is a "toolbox" of efficient practical methods for obtaining 3 dimensional (D) information; number, length, surface or volume from measurements made on $2 \mathrm{D}$ microscope sections (Haug, 1987; Osinubi, 2006). It relates the features seen on a section to the features in 3-D space in a well defined statistical way. Despite a formidable theoretical framework the methods are very easy to apply in practice.

Usually a three dimensional estimation of an organ describes numerical sampling methods for quantifying geometric parameters. This includes the number of cells, the length of a tube, the area of a particular region in a biological field, or the volume of 
Agric. Biol. J. N. Am., 2011, 2(11): 1390-1394

an organ. It therefore estimates the parameter of interest. The parameter can often be estimated from simple measurements of the samples, e.g. counting the number of cells (QuanToxPath, 1998). The stereological intention on the harvested testes would be to quantify the histological changes of the testes using scientifically proven relationships (Yama et al., 2011). Therefore the scope of the study covered by the present research applied simple stereological methods to assess the effect of three different doses of Momordica charantia (MC) seed extract on the testis of Sprague-Dawley rats. Our result would be a more accurate documentation of the effect of the extract on the testes.

\section{MATERIALS AND METHODS}

Procurement and identification of the plant material: The healthy fruit of $M$. charantia were purchased in a local market at Mushin Lagos State, Nigeria. The identification of the plant and fruit was done by a taxonomist in the Botany Department University of Ibadan. Specimens of the species were deposited in the Forest Herbarium of same Department, (voucher No. FHI 108422).

Processing and seed extraction: The whole fruit was dried in room temperature without exposure to sun light and the seeds extracted. Seeds were powdered and soxhleted with ethanol (95\%) as solvent for $24 \mathrm{~h}$ at the Pharmacognosy department, Faculty of Pharmacy, University of Lagos. The ethanolic extract was concentrated to dryness in a flash evaporator under reduced pressure and controlled temperature $\left(50-60^{\circ} \mathrm{C}\right)$ to obtain the dry crude extract. The percentage yield was $23.0 \% \mathrm{w} / \mathrm{w}$. The dry extract was stored in the refrigerator at $-4^{\circ} \mathrm{C}$ until needed for the treatment.

Preliminary screening tests for secondary metabolites: The preliminary tests, for the detection of secondary metabolites were carried out in ethanolic seed extract of MC by adopting standard methods (Figel, 1960; Gibbs, 1974). The extract (230 g) was dissolved in $1000 \mathrm{ml}$ of ethanol and filtered through Whatmann filter paper $\mathrm{N}^{\circ} 1$. Thus, the filtrate obtained was used as a test solution for the following preliminary screening tests like alkaloids, flavonoids, glycosides, lignin, phenols, saponins, sterols and tannins.

Animal model: A total of 20 adult sexually mature, healthy, colony-bred male rats of Sprague-Dawley strain, weighing 110-214 g were used for the experiment. The rats were housed randomly in polypropylene cages measuring 12 " x 10" x 8", under well-ventilated animal house conditions (ambient temperature: $28-31^{\circ} \mathrm{C}$, photoperiod: $12 \mathrm{~h}$ natural light and $12 \mathrm{~h}$ dark; relative humidity: $50-55 \%)$. The rats were given pelleted feed (Livestock feeds Plc. Ikeja, Lagos) and tap water ad libitum.

Experimental etiquette: The protocol of experimentation was approved by the Departmental Ethical Committee responsible for the use of laboratory Animals, University of Lagos, Nigeria. In a general meeting held on $19^{\text {th }}$ April, 2010, the work was reviewed and accepted to be in consistency with the standard of the use of laboratory animals (American Physiological Society, 2002).

The animals were divided into 4 groups (I-IV) consisting of five animals in each group. Groups I, II and III were treated daily with varying doses of the seed extract as follows: 15,25 and $50 \mathrm{mg} / 100 \mathrm{~g}$ body weight respectively. Group IV were the control rats used to compare events in the other groups. They received $5 \mathrm{ml} /$ day of physiological saline orally.

Computation of graded doses of dry extract and schedule of necropsy: Appropriate aliquots from the crude extract suspended in distilled water were administered based on the animal's individual body weight. These were calculated by simple proportion and given orally everyday between $13.00-16.00 \mathrm{~h}$ using an intra-gastric metal catheter. The treatment duration covered one spermatogenic cycle in rat equivalent to 56 days (Jegou et al., 2002). On the 57 th day, after $24 \mathrm{~h}$ of last dosing all the animals were weighed and sacrificed by cervical dislocation. This was followed by a ventral laparotomy to deliver the testes per abdomen.

Testicular gravimetry and algorithm of histometric parameters: The dissected testes were immediately separated out from the adherent tissue and weighed to the nearest $\mathrm{mg}$ on an electronic balance (Yama et al., 2010). Testicular volumes were estimated by water displacement method using Archimedes principle (Acott, 1999). The two testes of each rat were measured and the average value regarded as one observation. Tissues were processed for histometric analysis.

For each testis, seven perpendicular sections from the polar and the equatorial regions were sampled (Qin and Long, 2002) and an unbiased numerical estimation of the morphometric parameters was determined using a systematic random scheme (Gundersen and Jensen, 1987). The seven vertical sections per testis were selected by a systematic sampling method that ensured fair distribution 
Agric. Biol. J. N. Am., 2011, 2(11): 1390-1394

between the polar and equatorial regions of each testis. Briefly, a section was taken at the equator of each testis; one on each side of the equator, threequarters of the distance between the pole and the equator; another half-way between each pole and the equator; and one on each side of the equator, a quarter of the distance from each of the poles. For each stereological parameter $(D, A C, N A, N V, V V$, and $A V$ ), five randomly selected fields from all the seven sections of a single testis were viewed, and an estimation on each was carried out. The average from a total of seventy readings from five fields in seven sections of the two testes of one rat was obtained and this was recorded as one observation. Estimation of volume density of testicular components, diameter and number of seminiferous tubules were done on a screen on which slides were projected from a light microscope.

Diameter (D) and cross-sectional area (AC) of the seminiferous tubules: Circular or elliptical tubule outline with clear lumens were sampled using a test frame according to the unbiased forbidden-line rule. The diameters were then measured (Gundersen and Jensen, 1987). The diameter of seminiferous tubules with profiles that were round or nearly round were measured for each animal and the mean $D$ determined by taking the average of two diameters at right angles, D1 and D2. Data were taken only when D1 / D2 $\geq 0.85(1.0=$ a perfect circle). This is to eliminate longitudinal profiles which might exhibit different degrees of damage along their length and/or show irregular shrinkage (Osinubi, 2006). The cross sectional areas of the seminiferous tubules were determined from the formula $\mathrm{AC}=\mathrm{D} 2 \mathrm{X} \pi / 4$ (where $\pi$ is equivalent to 3.142 and $D$ is the mean diameter of the seminiferous tubules).

Number of profiles of seminiferous tubules in a unit area of testis (NA): The number of profiles of seminiferous tubules per unit area was determined by using the unbiased counting frame proposed by Gundersen in (1977). Using this frame, we counted all profiles that were completely inside or partly inside the frame provided they did not touch or intercept the forbidden line (full-drawn line) or exclusion edges or their extension.

\footnotetext{
Numerical and length densities (NV and LV) of the seminiferous tubules: This is the number of profiles per unit volume and was determined by using the modified Floderus equation: $N V=N A /(D+T)$ (Gilliland et al., 2001); where NA is the number of profiles per unit area $D$ the diameter and $T$ the average thickness of the section. The length density
}

which is the length of profile per tissue volume computed using the equation, $2 \times \mathrm{NA}$.

Statistical analysis: The data were statistically analyzed and expressed as Mean \pm SD. Analysis was carried out using analysis of variance (ANOVA) with Scheffe's post hoc test. The level of significance was considered at $p<0.05$.

\section{RESULTS}

The testicular density defined as weight in grams per unit volume in millilitres of the rat testes gave a mean equivalent to one which followed the same pattern in all the groups (Table 1). The mean seminiferous tubular diameters of rats treated with 15,25 , and $50 \mathrm{mg} / 100 \mathrm{~g}$ were: $210.10 \pm 9.89,86.18 \pm 4.31$ and $73.12 \pm 2.32 \mu \mathrm{m}$. These values showed a significant decline $(p<0.05)$ compared to $233.30 \pm 6.24 \mu \mathrm{m}$ of control rats. The variation in the cross sectional area of the seminiferous tubules follows a similar pattern as the tubular diameter (Table 2). Conversely there was a dose dependent increase in the mean number of profiles per unit area, length density and numerical density of seminiferous tubules of rats compared to their control counterpart (Table 2).

\section{DISCUSSION}

Deploying a stereological approach to quantifying histological sections helps to combat some fundamental issues associated with qualitative microscopic analysis. Almost all scientific laboratories are equipped with at least one light microscope and is probably the most ubiquitous of all scientific instruments. It has become a universally recognized symbol of scientific research. The amount of material that is actually examined in a light microscope is often a tiny fraction of the whole object of interest. Also the act of taking a thin histological section is both destructive and results in reduction of the dimensionality of the geometrical information obtained (QuanToxPath, 1998). In this study quantification was carried out on randomly sampled vertical histological sections using stereological tools in order to promote accuracy and eliminate these biases. 
Agric. Biol. J. N. Am., 2011, 2(11): 1390-1394

Table 1: Seminiferous tubular diameter, number of profiles per unit area and testicular weight and volume in experimental and control Sprague-Dawley rats

\begin{tabular}{|c|l|l|l|l|l|}
\hline Groups (n=20) & Group detail & $\mathbf{D}(\boldsymbol{\mu m})$ & $\mathbf{N}_{\mathbf{A}}\left(\mathbf{x} \mathbf{1 0}^{-8} \boldsymbol{\mu m}^{-\mathbf{2}}\right)$ & Weight (gm) & Volume (m) \\
\hline A & $15 \mathrm{mg} / 100 \mathrm{~g} \mathrm{b.w}$ & $210.10 \pm 9.89$ & $40.50 \pm 19.74$ & $0.90 \pm 0.08$ & $0.91 \pm 0.08$ \\
\hline B & $25 \mathrm{mg} / 100 \mathrm{~g} \mathrm{b.w}$ & $86.18 \pm 4.31$ & $101.75 \pm 4.99$ & $0.56 \pm 0.30$ & $0.57 \pm 0.31$ \\
\hline C & $50 \mathrm{mg} / 100 \mathrm{~g} \mathrm{b.w}$ & $73.12 \pm 2.32$ & $94.00 \pm 28.13$ & $0.32 \pm 0.27$ & $0.33 \pm 0.29$ \\
\hline D & & & & $1.14 \pm 0.22$ & $1.14 \pm 0.23$ \\
\hline
\end{tabular}

All values are expressed as Mean \pm Standard deviation; $p<0.05$; D: diameter of seminiferous tubule and $\mathrm{N}_{\mathrm{A}}$ : number of profiles per unit area of seminiferous tubule; b.w: body weight of rat

Table 2: The cross sectional area, numerical and length densities of seminiferous tubules in experimental and control Sprague-Dawley rats

\begin{tabular}{|c|c|c|c|c|}
\hline Groups ( $n=20$ ) & Group detail & $\begin{array}{c}\boldsymbol{A}_{c} \\
\left(\times 10^{3} \boldsymbol{\mu m}^{2}\right)\end{array}$ & $\begin{array}{c}N_{V} \\
\left(\times 10^{-10} \mu^{-3}\right)\end{array}$ & $\begin{array}{c}L v \\
\left(x 10^{-8} \mu m^{-2}\right)\end{array}$ \\
\hline $\boldsymbol{A}$ & 15 mg/100 g b.w & $34.73 \pm 3.19$ & $18.75 \pm 0.10$ & $81.00 \pm 39.48$ \\
\hline$B$ & $25 \mathrm{mg} / 100 \mathrm{~g}$ b.w & $5.84 \pm 0.58$ & $111.75 \pm 0.09$ & $203.50 \pm 9.98$ \\
\hline C & $50 \mathrm{mg} / 100 \mathrm{~g}$ b.w & $4.20 \pm 0.27$ & $120.60 \pm 0.36$ & $188.00 \pm 56.27$ \\
\hline $\bar{D}$ & Physiological saline & $42.43 \pm 2.88$ & $11.20 \pm 0.03$ & $52.00 \pm 14.21$ \\
\hline
\end{tabular}

All values are expressed as Mean \pm Standard deviation; $p<0.05$; $A_{c}$ : cross sectional area, $N_{v}$ : numerical density and Lv: length density of seminiferous tubules; b.w: body weight of rat

The results showed a dose dependent decrease in testicular weight and volume, the luminal diameter and cross-sectional area of seminiferous tubules. On the other hand there was an increase in the number of profiles per unit area, length density and numerical density of the seminiferous tubules. These estimates imply that the regular morphologies of the seminiferous epithelium, responsible for the exceptional formation of the luminal fluid, have been perturbed (Okanlawon and Ashiru, 1998). Researchers have shown that the germinal epithelium is solely accountable for the production of luminal fluid an indispensable precondition for spermatogenesis (Jegou, 1992; Fisher, 2002; Syed and Hecht, 2002). This suggests that the extract in a dose related manner compromised the process of spermatogenesis as reflected by a reduction the in tubular lumen. The qualitative study of the antifertility effect of MC using similar graded doses of the seed extract supports these present quantitative estimates of testicular responses (Yama et al., 2011). It was shown to suppress both testicular testosterone and sperm production in albino rats.

The estimation of the specific gravity of testis; a subset of the weight/volume by researchers has shown it to be almost equal to one (Mori and Christensen, 1980).This is in tandem with our findings because testicular volume and weight quantities in these experiments were more or less the same and both parameters were equally dose dependently distorted.

The level of testicular testosterone correlates positively with the Leydig cell number and size of the interstitium of testes (Castro et al., 2002). The dose dependent reduction in the testicular volume and diameter of the tubules from our study would only suggest a significant reduction in the interstitium due to the destruction of the Leydig cells. These cells are endocrine cells responsible for the production of testosterone. Our previous study supported depleted testosterone (Yama et al., 2011).

It is not completely likely to forecast the expected corollary of these altered geometric associations on tubular sperm production or flow because the present morphometric data forms only part of the complete depiction. Other factors are clearly important. These include number of spermatogenic cells in the basal compartment and the Sertoli-Sertoli cell barrier which determines the number of cells in the adluminal compartment (Osinubi, 2006).

However the stereological deductions obtained from the microscopic observations in this study are actually a sound conclusion of the geometrical feature of the rat testis. This provides quantitative 
evidence that the seed extract of Momordica charantia at a dose of $50 \mathrm{mg} / 100 \mathrm{~g}$ produces greater histomorphometric alterations than 15 and $25 \mathrm{mg} / 100$ $\mathrm{g}$ in male rats. This corresponds similarly to the qualitative testicular responses we studied earlier.

\section{ACKNOWLEDGEMENT}

I wish to acknowledge $\mathrm{Mr}$ Adeleke of the Pharmacognosy Department Faculty of Pharmacy University of Lagos Nigeria for his assistance with the preparation of the herbal decoction and also support of this work. Also special thanks goes to Dr. Dolapo Duro Consultant physician and biostatistician College of Medicine University of Lagos Nigerian for statistical analysis of the work.

\section{REFERENCES}

Acott C. The diving "Law-ers": A brief resume of their lives. South Pacific Underwater Medicine Society Journal, 1999; 29 (1).

American Physiological Society. Guiding principles for research involving animals and human beings. Am J Physiol Regul Integr Comp Physiol, 2002; 283: 281-283.

Castro AC, Berndtson AW, Cardoso FM. Plasma and testicular testosterone level, $\mathrm{V} v$ and number of Leydig cells and spermatogenic efficiency of rabbits. Braz $J$ Med Biol Res, 2002; 35: 493.

Figel F. Spot test in organic analysis. London, Elsevier Publications Co., 1960; p.143.

Fisher D. New light shed on fluid formation in seminiferous tubules of the rat. J Physio, 2002; 542, 445-452.

Gibbs RD. Chemotaxonomy of flowering plants. Montreal, McGill Queen's University Press, 1974; pp.523-619.

Gilliland KO, Freel CD, Lane CW, Fowler WC, Costello NJ. Multilamellar bodies as potential scattering particles in human age-related nuclear cataracts. Molecular vision, 2001; 7: 120-130.

Gundersen HJG, Jensen EB. The efficiency of systematic sampling in stereology and its predictions. J Microsc 1987; 147: 229-263.

Gundersen HJG. Notes on the estimation of the numerical density of arbitrary profiles: The edge effect. $J$ Micros 1977; 111: 21-23.
Haug $H$. Brain sizes, surfaces, and neuronal sizes of the cortex cerebri: A stereological investigation of Man and his variability and a comparison with some mammals (primates, whales, marsupials, insectivores, and one elephant). Am J Anatomy. 1987; 180: 126-142

Howard CV, Reed MG. Unbiased stereology $2^{\text {nd }}$ Edition 2005 pp 103-104

Jegou B, Pineau C, Toppari J. Spermatogenesis in-vitro in mammals. Assisted Reproductive Technology: Accomplishments and New Horizons. Cambridge: Cambridge University Press, 2002; 1: 3-60.

Jegou B. The Sertoli cell. Baillier's Clin Endocrinol Metab, 1992; 6, 273-311.

Mori $\mathrm{H}$, Christensen AK. Morphometric analysis of Leydig cells in the normal rat testis. J Cell Res, 1980; 84, 340-354.

Okanlawon AO, Ashiru OA. Sterological estimation of seminiferous tubular dysfunction in chloroquine treated rats. Afr J Med Med Sci, 1998; 27, 101-106.

Osinubi AA. Quinine-induced cytoarchitectural and morphometric alterations in the testes of SpragueDawley rats (Ph.D. Thesis). Lagos: University of Lagos, 2006; pp. 1-18.

Qin B, Long MA. Morphometric study on Leydig cells in capsulotomized testis of rats. Asian J Androl, 2002; 4, 49-53.

QuanToxPath (QuantitativeToxico-Pathology) 1998. Quantitative Microscopy http://www.liv.ac.uk/fetoxpath/quantmic.htm

Syed V, Hecht NB. Disruption of germ cell-Sertoli cell interactions leads to spermatogenic defects. Mol Cell Endocrinol, 2002; 186, 155-157.

Wong PYD. Do seminiferous tubules secrete a fluid rich in $\mathrm{KHCO}_{3}$ ? J Physiol, 2002; 542, 335-336.

Yama OE, Duru FI, Oremosu AA, Osinubi AA, Norohna CC, Okanlawon AO. Sperm quotient in SpragueDawley rats fed graded doses of seed extract of Momordica charantia. Middle East Fertility Society Journal, 2011; Vol. 16, Issue 2, Pg. 154-158.

Yama OE, Osinubi AA, Noronha CC, Okanlawon AO. Effect of methanolic seed extract Momordica charantia on body weight and serum cholesterol level of male Sprague-Dawley rats. Nigerian quarterly journal of Hospital Medicine, 2010; Vol. 20 (4): 209-213 